MODELING, IDENTIFICATION AND CONTROL, 1996, vOL. 17, NO. 2, 153-165

doi:10.4173/mic.1996.27

\title{
Identification of dynamically positioned ships
}

\author{
THOR I. FOSSEN $\dagger$, SVEIN I. SAGATUN $\ddagger$ \\ and ASGEIR J. SØRENSEN§
}

Keywords: Dynamic positioning, identification, full-scale sea trials, Kalman filtering, self-tuning control, marine systems

\begin{abstract}
Todays model-based dynamic positioning (DP) systems require that the ship and thruster dynamics are known with some accuracy in order to use linear quadratic optical control theory. However, it is difficult to identify the mathematical model of a dynamically positioned (DP) ship since the ship is not persistently excited under DP. In addition the ship parameter estimation problem is nonlinear and multivariable with only position and thruster state measurements available for parameter estimation. The process and measurement noise must also be modeled in order to avoid parameter drift due to environmental disturbances and sensor failure. This article discusses an off-line parallel extended Kalman filter (EKF) algorithm utilizing two measurement series in parallel to estimate the parameters in the DP ship model. Full-scale experiments with a supply vessel are used to demonstrate the convergence and robustness of the proposed parameter estimator.
\end{abstract}

\section{Introduction}

Modern dynamic positioning (DP) systems are based on model-based feedback control. The state estimator and control law are designed by applying a low-frequency (LF) mathematical model of the ship motions caused by currents, wind and 2nd-order wave loads, and high-frequency (HF) model of the 1st-order ship motions caused by 1st-order wave disturbances; see Fossen (1994).

Model-based control systems utilizing stochastic optimal control theory and Kalman filtering techniques was first employed with the DP problem by Balchen et al. (1976). Later extensions and modifications of this work have been reported by Balchen et al. (1980a, 1980b), Grimble et al. (1980a, 1980b), Fung and Grimble (1983) and Sælid et al. (1983).

In order to achieve good performance of the control system it is necessary to have a sufficient detailed mathematical model of the ship. ABB Industri AS in Oslo has marketed a new self-tuning model-based DP system based on the results presented in this article whereas the control system design is discussed in Sørensen et al. (1995).

Received 20 August 1995.

†The Norwegian Institute of Technology, Department of Engineering Cybernetics, N-7034 Trondheim, Norway. E-mail: tif@itk.unit.no.

$\ddagger$ ABB Industri AS, Hasleveien 50, P.O. Box 6540 Rodeløkka, N-0501 Oslo, Norway. E-mail: sis@ttsinit.no

§ ABB Industri AS, Hasleveien 50, P.O. Box 6540 Rodeløkka, N-0501 Oslo, Norway. E-mail: Asgeir.Sorensen@noina.abb.telemax.no

Reprinted from Control Engineering Practice, 1996. with kind permission from Elsevier Science I td, Oxford, UK 


\section{Ship and thruster models}

This section describes the mathematical model of the thrusters and the LF motion of the ship.

\subsection{Thruster model}

Most DP ships use thrusters and main propellers to maintain their position and heading. The thrust force of a pitch-controlled thruster can be approximated by

$$
F(n, p)=K(n)\left|p-p_{0}\right|\left(p-p_{0}\right)
$$

where the force coefficient $K(n)$ is assumed to be constant for constant propeller revolution $n, P$ is the 'traveled distance per revolution', $D$ is the propeller diameter and

$$
p=P / D
$$

is the pitch ratio. $p_{0}$ is pitch ratio off-set defined such that $p=p_{0}$ yields zero thrust, that is

$$
F\left(n, p_{0}\right)=0
$$

Thrust forces and moment. The thrust forces and moment vector $\tau \in \mathbb{R}^{3}$ (surge, sway and yaw) for the supply vessel in Fig. 1 can be written

$$
\tau=\boldsymbol{T K u}
$$

where $\boldsymbol{u} \in \mathbb{R}^{r}$ is a control variable defined as

$$
\boldsymbol{u}=\left[\left|p_{1}-p_{10}\right|\left(p_{1}-p_{10}\right),\left|p_{2}-p_{20}\right|\left(p_{2}-p_{20}\right), \ldots,\left|p_{r}-p_{r 0}\right|\left(p_{r}-p_{r 0}\right)\right]^{\mathrm{T}}
$$

where $p_{i 0}(i=1 \ldots r)$ are the pitch ratio off-sets for thruster no. $i$ and $r$ is the maximum number of thrusters.

Thrust force coefficient matrix. The thrust force coefficient matrix $\boldsymbol{K}$ is a diagonal matrix of thrust force coefficients defined as

$$
\boldsymbol{K}=\operatorname{diag}\left\{K_{1}\left(n_{1}\right), K_{2}\left(n_{2}\right), \ldots, K_{r}\left(n_{r}\right)\right\}
$$

where $n_{i}(i=1 \ldots r)$ is the propeller revolution of propeller number $i$. The thrust forces $K_{i}\left(n_{i}\right) u_{i}$ are distributed to the surge, sway and yaw modes by a $3 \times r$ thruster configuration matrix $\boldsymbol{T}$.

Thruster configuration matrix. Consider the ship in Fig. 1 which is equipped with two main propellers, three tunnel thrusters and one azimuth thruster which can be rotated to an arbitrary angle $\alpha$. The control variables are assigned according to

$$
\begin{aligned}
& u_{1}=\text { port main propeller } \\
& u_{2}=\text { starboard main propeller } \\
& u_{3}=\text { aft tunnel thruster I }
\end{aligned}
$$$$
u_{4}=\text { aft tunnel thruster II }
$$$$
u_{5}=\text { bow tunnel thruster }
$$$$
u_{6}=\text { bow azimuth thruster }
$$ 


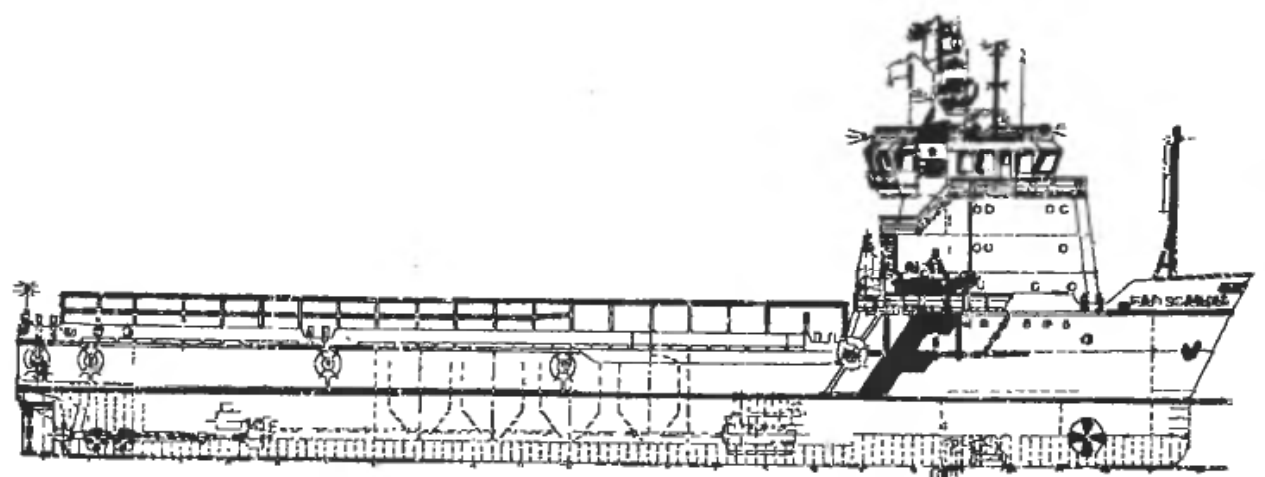

Figure 1. Picture showing the supply vessel which was used during the sea trials in the North Sea $(L=76 \cdot 2 \mathrm{~m})$.
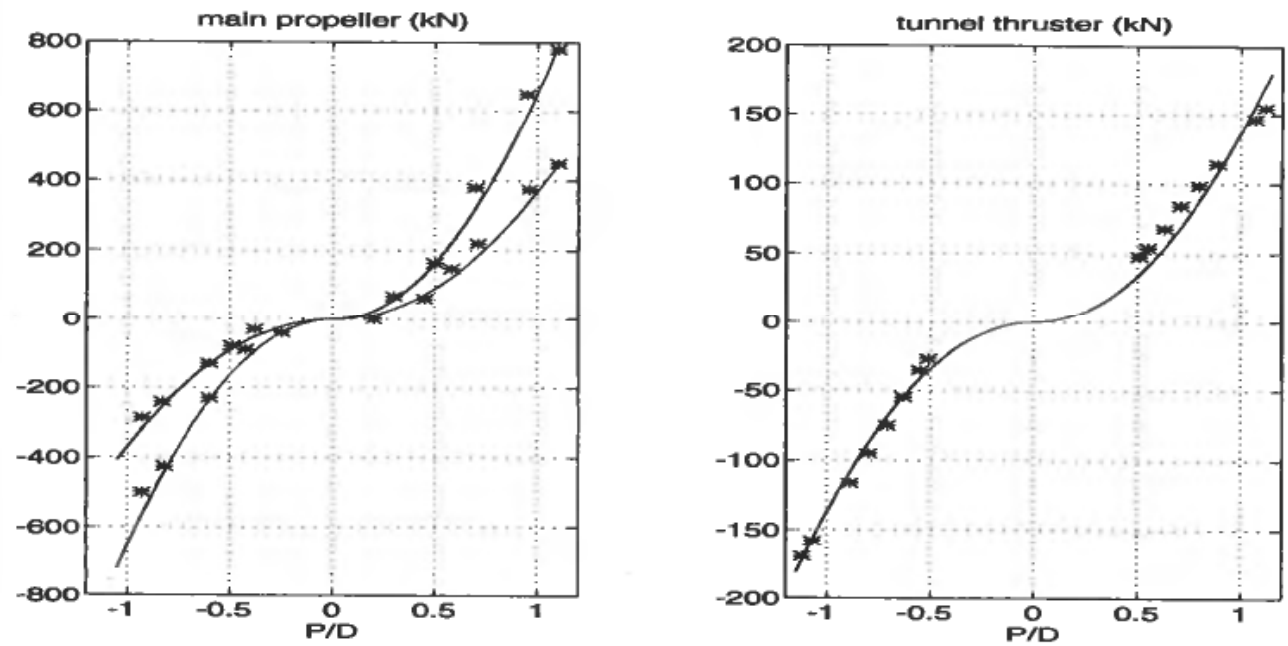

Figure 2. Experimentally measured thrust (asterisks) and thruster model approx. Eqn. (1) versus $p=P / D$. Left plot: $F(122, p)=370 p|p|$ and $F(160, p)=655 p|p|$. Right plot: $F(236, p)=137 p|p|$. Propeller revolution is in rpm.

The following thruster configuration matrix is obtained

$$
\boldsymbol{T}=\left[\begin{array}{cccccc}
1 & 1 & 0 & 0 & 0 & \cos \alpha \\
0 & 0 & 1 & 1 & 1 & \sin \alpha \\
l_{1} & -l_{2} & -l_{3} & -l_{4} & l_{5} & l_{6} \sin \alpha
\end{array}\right]
$$

where $l_{i}(i=1 \ldots 6)$ are the moment arms in yaw. It is also seen that $l_{2}=l_{1}$ (symmetrical location of main propellers). The thrust demands are defined such that positive thrust force/moment results in positive motion according to the vessel parallel axis system defined such that positive $x$-direction is forwards, positive $y$-direction is starboard and positive $z$-direction is downwards. The origin is located in the centre of buoyancy. 


\section{2. $L F$ ship dynamics}

The LF ship model in surge, sway and yaw can be described by Fossen (1994)

$$
M \dot{v}+C(v) v+D\left(v-v_{c}\right)=\tau+w
$$

where $v=[u, v, r]^{\mathrm{T}}$ denotes the $\mathrm{LF}$ velocity vector, $v_{c}=\left[u_{c}, v_{c}, r_{c}\right]^{\mathrm{T}}$ is a vector of current velocities, $\tau$ is a vector of control forces and moments and $\boldsymbol{w}=\left[w_{1}, w_{2}, w_{3}\right]^{\mathrm{T}}$ is a vector of zero-mean Gaussian white noise processes describing unmodeled dynamics and disturbances. Notice that $r_{c}$ does not represent a physical current velocity, but can be interpreted as the effect of currents in yaw. The current states are useful in the parameter estimator since they represent slowly-varying non-zero bias terms.

The inertia matrix including hydrodynamic added mass terms is assumed to be positive definite $\boldsymbol{M}=\boldsymbol{M}^{\mathrm{T}}>\mathbf{0}$ for a dynamically positioned ship, whereas $\boldsymbol{D}>\boldsymbol{0}$ is a strictly positive matrix representing linear hydrodynamic damping. Nonlinear damping is assumed to be negligible for station-keeping of ships whereas the assumption of starboard and port symmetry implies that $\boldsymbol{M}$ and $\boldsymbol{D}$ can be written

$$
\begin{aligned}
\boldsymbol{M} & =\left[\begin{array}{ccc}
m-X_{\dot{u}} & 0 & 0 \\
0 & m-Y_{\dot{v}} & m x_{G}-Y_{\dot{r}} \\
0 & m x_{G}-Y_{\dot{r}} & I_{z}-N_{\dot{r}}
\end{array}\right] \\
\boldsymbol{D} & =\left[\begin{array}{ccc}
-X_{u} & 0 & 0 \\
0 & -Y_{v} & -Y_{r} \\
0 & -N_{v} & -N_{r}
\end{array}\right]
\end{aligned}
$$

The Coriolis and centrifugal matrix $\boldsymbol{C}(\boldsymbol{v})$ is included in the model to improve the convergence of the parameter estimator. Moreover this matrix may be significant for a ship operating at some speed whereas $\boldsymbol{C}(\boldsymbol{v})=\mathbf{0}$ for a ship at rest. It should be noted that inclusion of $\boldsymbol{C}(v)$ in the model will not increase the number of parameters to be estimated sine $C(v)$ only is a function of the elements $m_{i j}$ of the inertia matrix; see Theorem 2.2. on p. 27 in Fossen (1994). In fact $\boldsymbol{M}=\left\{m_{i j}\right\}$ yields

$$
\boldsymbol{C}(\boldsymbol{v})=\left[\begin{array}{ccc}
0 & 0 & -m_{22} v-m_{23} r \\
0 & 0 & m_{11} u \\
m_{22} v+m_{23} r & -m_{11} u & 0
\end{array}\right]
$$

where the non-zero elements $m_{i j}=-m_{j i}$ are defined according to (9) such that

$$
\begin{array}{ll}
m_{11}=m-X_{\dot{u}} & m_{23}=m x_{G}-Y_{\dot{r}} \\
m_{22}=m-Y_{\dot{v}} & m_{33}=I_{z}-N_{\dot{r}}
\end{array}
$$

\subsection{Kinematics}

The kinematic equation of motion for a ship is

$$
\dot{\boldsymbol{\eta}}=\boldsymbol{J}(\boldsymbol{\eta}) \boldsymbol{v}
$$




\begin{tabular}{|c|c|}
\hline $\begin{array}{l}\text { System model } \\
\text { Measurement }\end{array}$ & $\begin{array}{l}\boldsymbol{\xi}(k+1)=\mathscr{F}(\xi(k), \boldsymbol{u}(k))+\boldsymbol{\Gamma} \boldsymbol{w}(k) ; \quad \boldsymbol{w}(k) \sim(\mathbf{0}, \boldsymbol{Q}(k)) \\
\boldsymbol{z}(k)=\mathscr{H}(\xi(k))+\boldsymbol{v}(k) ; \quad \boldsymbol{v}(k) \sim N(\mathbf{0}, \boldsymbol{R}(k))\end{array}$ \\
\hline Initial conditions & $\hat{\xi}(0)=\xi_{0}$ \\
\hline $\begin{array}{l}\text { State estimate propagation } \\
\text { Error covariance propagation }\end{array}$ & $\begin{array}{l}\bar{\xi}(k+1)=\mathscr{F}(\hat{\xi}(k), \boldsymbol{u}(t)) \\
\overline{\boldsymbol{P}}(k+1)=\boldsymbol{\Phi}(k) \hat{\boldsymbol{P}}(k) \boldsymbol{\Phi}^{\mathrm{T}}(k)+\boldsymbol{\Gamma}(k) Q(k) \Gamma^{\mathrm{T}}(k)\end{array}$ \\
\hline $\begin{array}{l}\text { Gain matrix } \\
\text { State estimate update } \\
\text { Error covariance update }\end{array}$ & $\begin{array}{l}\boldsymbol{K}(k)=\overline{\boldsymbol{P}}(k) \boldsymbol{H}^{\mathrm{T}}(k)\left[\boldsymbol{H}(k) \overline{\boldsymbol{P}}(k) \boldsymbol{H}^{\mathrm{T}}(k)+\boldsymbol{R}(k)\right]^{-1} \\
\hat{\xi}(k)=\bar{\xi}(k)+\boldsymbol{K}(k)[z(k)-\mathscr{H}(\bar{\xi}(k))] \\
\hat{\boldsymbol{P}}(k)=[\boldsymbol{I}-\boldsymbol{K}(k) \boldsymbol{H}(k)] \overline{\boldsymbol{P}}(k)[\boldsymbol{I}-\boldsymbol{K}(k) \boldsymbol{H}(k)]^{\mathrm{T}}+\boldsymbol{K}(k) \boldsymbol{R}(k) \boldsymbol{K}^{\mathrm{T}}(k)\end{array}$ \\
\hline Definitions & $\Phi(k)=\left.\frac{\partial \mathscr{F}(\cdot)}{\partial \xi(k)}\right|_{\xi(k)=\hat{\xi}(k)}$ \\
\hline
\end{tabular}

Table 1. Summary of discrete-time extended Kalman filter (EKF).

where $\boldsymbol{\eta}=[x, y, \psi]^{\mathrm{T}}$ and $\boldsymbol{J}(\boldsymbol{\eta})$ is a rotation matrix defined as

$$
\boldsymbol{J}(\boldsymbol{\eta})=\left[\begin{array}{ccc}
\cos \psi & -\sin \psi & 0 \\
\sin \psi & \cos \psi & 0 \\
0 & 0 & 1
\end{array}\right]
$$

\section{Off-line parameter estimator}

The off-line parameter estimator is based on the state augmented extended Kalman filter (EKF).

\subsection{State augmented extended Kalman filter}

Consider the following nonlinear system

$$
\begin{aligned}
& \boldsymbol{x}(k+1)=\boldsymbol{f}(\boldsymbol{x}(k), \boldsymbol{u}(k), \boldsymbol{\theta}(k))+\boldsymbol{w}_{1}(k) \\
& \boldsymbol{\theta}(k+1)=\boldsymbol{\theta}(k)+\boldsymbol{\eta}(k)
\end{aligned}
$$

where $\boldsymbol{x} \in \mathbb{R}^{n}$ is the state vector, $\boldsymbol{u} \in \mathbb{R}^{r}$ is the input vector, $\boldsymbol{\theta} \in \mathbb{R}^{p}$ is the unknown parameter vector to be estimated and $\boldsymbol{w}_{1}, \boldsymbol{\eta} \in \mathbb{R}^{n}$ are zero-mean Gaussian white noise processes. This model can be expressed in augmented state-space form as

$$
\xi(k+1)=\mathscr{F}(\xi(k), \boldsymbol{u}(k))+\boldsymbol{w}(k)
$$

where $\xi=\left[\boldsymbol{x}^{\mathrm{T}}, \boldsymbol{\theta}^{\mathrm{T}}\right]^{\mathrm{T}}$ is the augmented state vector, $\boldsymbol{w}=\left[\boldsymbol{w}_{1}^{\mathrm{T}}, \boldsymbol{\eta}^{\mathrm{T}}\right]^{\mathrm{T}}$ and

$$
\mathscr{F}(\xi(k), \boldsymbol{u}(k))=\left[\begin{array}{c}
\boldsymbol{f}(\boldsymbol{x}(k), \boldsymbol{u}(k), \boldsymbol{\theta}(k)) \\
\theta(k)
\end{array}\right]
$$

Furthermore, it is assumed that the measurement equation can be written

$$
z(k)=\mathscr{H}(\xi(k))+v(k)
$$

where $z \in \mathbb{R}^{m}$ and $m$ is the number of sensors. The discrete-time extended Kalman filter algorithm in Table 1 can then be applied to estimate $\xi=\left[x^{\mathrm{T}}, \theta^{\mathrm{T}}\right]^{\mathrm{T}}$ in (17) by means of the measurement (19). For details on the implementation issues see Gelb et al. (1988). 


\subsection{Off-line EKF for parallel processing}

In order to improve the convergence and performance of the parameter estimator the same quantity can be measured $N \geq 2$ times for different excitation sequences. Moreover, let the input $\boldsymbol{u}_{i} \in \mathbb{R}^{r}$ correspond to the state vector $\boldsymbol{x}_{i} \in \mathbb{R}^{n}$ and measurement vector $z_{i} \in \mathbb{R}^{m}$ for $(i=1 \ldots N)$. Under the assumption of constant parameters, the parameter vector $\boldsymbol{\theta} \in \mathbb{R}^{p}$ will be the same for all these subsystems. This can be expressed mathematically as

$$
\begin{aligned}
\boldsymbol{x}_{1}(k+1) & =\boldsymbol{f}\left(\boldsymbol{x}_{1}(k), \boldsymbol{u}_{1}(k), \boldsymbol{\theta}(k)\right)+\boldsymbol{w}_{1}(k) \\
\boldsymbol{x}_{2}(k+1) & =\boldsymbol{f}\left(\boldsymbol{x}_{2}(k), \boldsymbol{u}_{2}(k), \boldsymbol{\theta}(k)\right)+\boldsymbol{w}_{2}(k) \\
\vdots & \\
\boldsymbol{x}_{N}(k+1) & =\boldsymbol{f}\left(\boldsymbol{x}_{N}(k), \boldsymbol{u}_{N}(k), \boldsymbol{\theta}(k)\right)+\boldsymbol{w}_{N}(k) \\
\boldsymbol{\theta}(k+1) & =\boldsymbol{\theta}(k)+\boldsymbol{\eta}(k)
\end{aligned}
$$

with measurements

$$
\begin{aligned}
\boldsymbol{z}_{1}(k) & =\boldsymbol{h}_{1}\left(\boldsymbol{x}_{1}(k), \boldsymbol{\theta}(k)\right)+\boldsymbol{v}_{1}(k) \\
\boldsymbol{z}_{2}(k) & =\boldsymbol{h}_{2}\left(\boldsymbol{x}_{2}(k), \boldsymbol{\theta}(k)\right)+\boldsymbol{v}_{2}(k) \\
& \vdots \\
\boldsymbol{z}_{N}(k) & =\boldsymbol{h}_{N}\left(\boldsymbol{x}_{N}(k), \boldsymbol{\theta}(k)\right)+\boldsymbol{v}_{N}(k)
\end{aligned}
$$

Hence, this system can be written in augmented state-space form according to

$$
\begin{gathered}
\boldsymbol{x}(k+1)=\mathscr{F}(\boldsymbol{\xi}(k), \boldsymbol{u}(k))+\boldsymbol{w}(k) \\
z(k)=\mathscr{H}(\xi(k))+\boldsymbol{v}(k)
\end{gathered}
$$

where $\boldsymbol{u}=\left[\boldsymbol{u}_{1}^{\mathrm{T}}, \ldots, \boldsymbol{u}_{N}^{\mathrm{T}}\right]^{\mathrm{T}}, z=\left[z_{1}^{\mathrm{T}}, \ldots, z_{N}^{\mathrm{T}}\right]^{\mathrm{T}}, \xi=\left[x_{1}^{\mathrm{T}}, \ldots, \boldsymbol{x}_{N}^{\mathrm{T}}, \theta^{\mathrm{T}}\right]^{\mathrm{T}}$ and

$$
\begin{aligned}
\mathscr{F}(\xi(k), \boldsymbol{u}(k))=\left[\begin{array}{c}
\boldsymbol{f}\left(\boldsymbol{x}_{1}(k), \boldsymbol{u}_{1}(k), \boldsymbol{\theta}(k)\right) \\
\boldsymbol{f}\left(\boldsymbol{x}_{2}(k), \boldsymbol{u}_{2}(k), \boldsymbol{\theta}(k)\right) \\
\vdots \\
\boldsymbol{f}\left(\boldsymbol{x}_{N}(k), \boldsymbol{u}_{N}(k), \boldsymbol{\theta}(k)\right) \\
\boldsymbol{\theta}(k)
\end{array}\right] \\
\mathscr{H}(\xi(k))=\left[\begin{array}{c}
\boldsymbol{h}\left(\boldsymbol{x}_{1}(k), \boldsymbol{\theta}(k)\right) \\
\boldsymbol{h}\left(\boldsymbol{x}_{2}(k), \boldsymbol{\theta}(k)\right) \\
\vdots \\
\boldsymbol{h}\left(\boldsymbol{x}_{N}(k), \boldsymbol{\theta}(k)\right)
\end{array}\right]
\end{aligned}
$$

It is observed that $\operatorname{dim} x=N n+p, \operatorname{dim} u=N r$ and $\operatorname{dim} z=N m$. It is then evident that more information about the system is obtained by using multiple measurement sequences. Increased information improves parameter identifiability and reduces the possibility for parameter drift. However it should be noted that parallel processing implies that the parameters estimation must be performed off-line. 


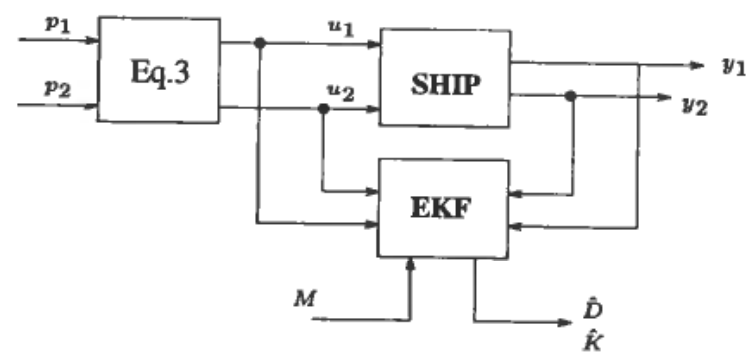

Figure 3. Parallel configuration of EKF for $N=2$.

For ship applications significant performance improvement is reported already for $N=2$; see Abkowitz (1975), Abkowitz (1980) and Hwang (1980).

\section{Identification of a supply vessel}

Full-scale experiments with the supply vessel in Fig. 1 will be used to demonstrate the convergence of the proposed parameter estimation algorithm.

\subsection{System identification model}

The system identification model is based on the mathematical model presented in Section 2. Assuming no environmental disturbances a dynamically positioned ship can be described by the following non-dimensional model (Bis-system) in surge, sway and yaw (see p. 178 in Fossen 1994)

$$
M^{\prime \prime} \dot{v}^{\prime \prime}+C^{\prime \prime}\left(v^{\prime \prime}\right) v^{\prime \prime}+D^{\prime \prime} v^{\prime \prime}=T^{\prime \prime} K^{\prime \prime} u^{\prime \prime}
$$

where

$$
\begin{aligned}
\boldsymbol{M}^{\prime \prime} & =\left[\begin{array}{ccc}
1-X_{u}^{\prime \prime} & 0 & 0 \\
0 & 1-Y_{\dot{v}}^{\prime \prime} & x_{G}^{\prime \prime}-Y_{\dot{r}}^{\prime \prime} \\
0 & x_{G}^{\prime \prime}-Y_{\dot{r}}^{\prime \prime} & k_{z}^{2}-N_{\dot{r}}^{\prime \prime}
\end{array}\right] \\
\boldsymbol{D}^{\prime \prime} & =\left[\begin{array}{ccc}
-X_{u}^{\prime \prime} & 0 & 0 \\
0 & -Y_{v}^{\prime \prime} & -Y_{r}^{\prime \prime} \\
0 & -N_{v}^{\prime \prime} & -N_{r}^{\prime \prime}
\end{array}\right]
\end{aligned}
$$

The thruster configuration matrix was computed to be

$$
\boldsymbol{T}^{\prime \prime}=\left[\begin{array}{lcccll}
1.0000 & 1.0000 & 0 & 0 & 0 & 0 \\
0 & 0 & 1.0000 & 1.0000 & 1.0000 & 1.0000 \\
0.0472 & -0.0472 & -0.4108 & -0.3858 & 0.4554 & 0.3373
\end{array}\right]
$$

whereas $\boldsymbol{K}^{\prime \prime}=\operatorname{diag}\left\{K_{1}^{\prime \prime}, K_{2}^{\prime \prime}, K_{3}^{\prime \prime}, K_{4}^{\prime \prime}, K_{5}^{\prime \prime}, K_{6}^{\prime \prime}\right\}$ is the unknown matrix to be estimated. In addition to this it will be assumed that $\boldsymbol{D}^{\prime \prime}$ is unknown. An a priori estimate of $\boldsymbol{M}^{\prime \prime}$ is calculated by applying semi-empirical methods. For more details about the computation 


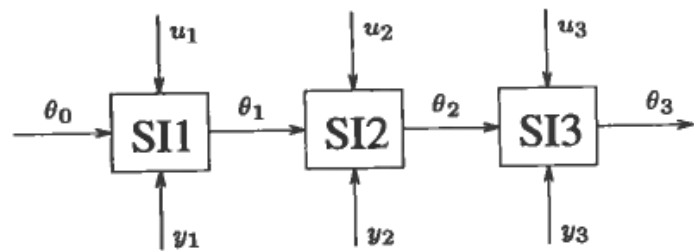

Figure 4. Decoupled parameter estimation in terms of three system identification schemes SI1-SI3.
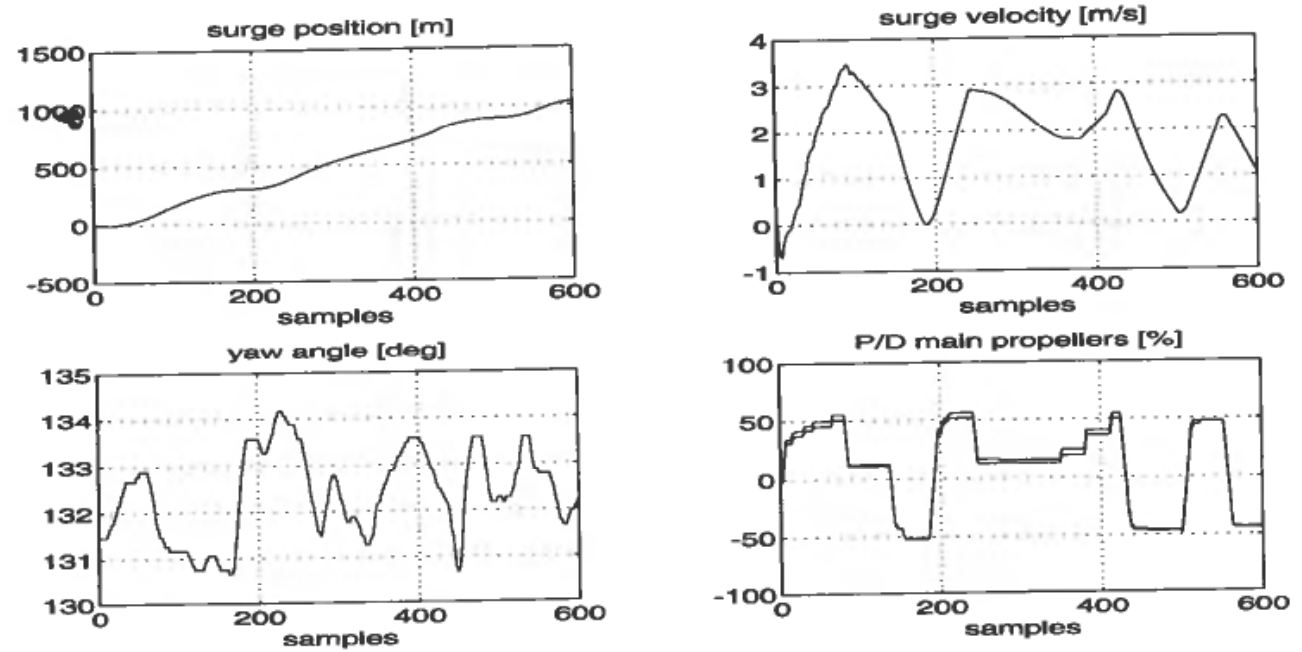

Figure 5. Sea Trial 1: Full scale experiment with a supply vessel (uncoupled surge).

of the added mass derivatives $X_{\dot{u}}^{\prime \prime}, Y_{\dot{v}}^{\prime \prime}, N_{\dot{r}}^{\prime \prime}$ and $Y_{\dot{r}}^{\prime \prime}$, see Faltinsen (1990). The inertia matrix $\boldsymbol{M}^{\prime \prime}$ was computed to be

$$
\boldsymbol{M}^{\prime \prime}=\left[\begin{array}{lcc}
1.1274 & 0 & 0 \\
0 & 1.8902 & -0.0744 \\
0 & -0.0744 & 0.1278
\end{array}\right]
$$

Hence $\boldsymbol{K}^{\prime \prime}$ and $\boldsymbol{D}^{\prime \prime}$ are the only remaining unknown matrices in the DP model (26).

\subsection{Sea trials}

In order to improve the convergence of the parameter estimator it is proposed to use several off-line measurement series generated by a number of carefully predefined manoeuvres. For instance, it is advantageous to decouple the surge mode from the sway and yaw modes in order to improve the convergence of the parameter estimator. This is motivated by the block diagonal structure of $M^{\prime \prime}$ and $D^{\prime \prime}$.

Decoupled ship manoeuvres. The following three decoupled ship manoeuvres are proposed:

(1) uncoupled surge: the ship is only allowed to move in surge (constant heading) by means of the main propellers $u_{1}$ and $u_{2}$. The heading is controlled by means of one of the bow thrusters. At least two manoeuvres should be performed; see Fig. 5. 

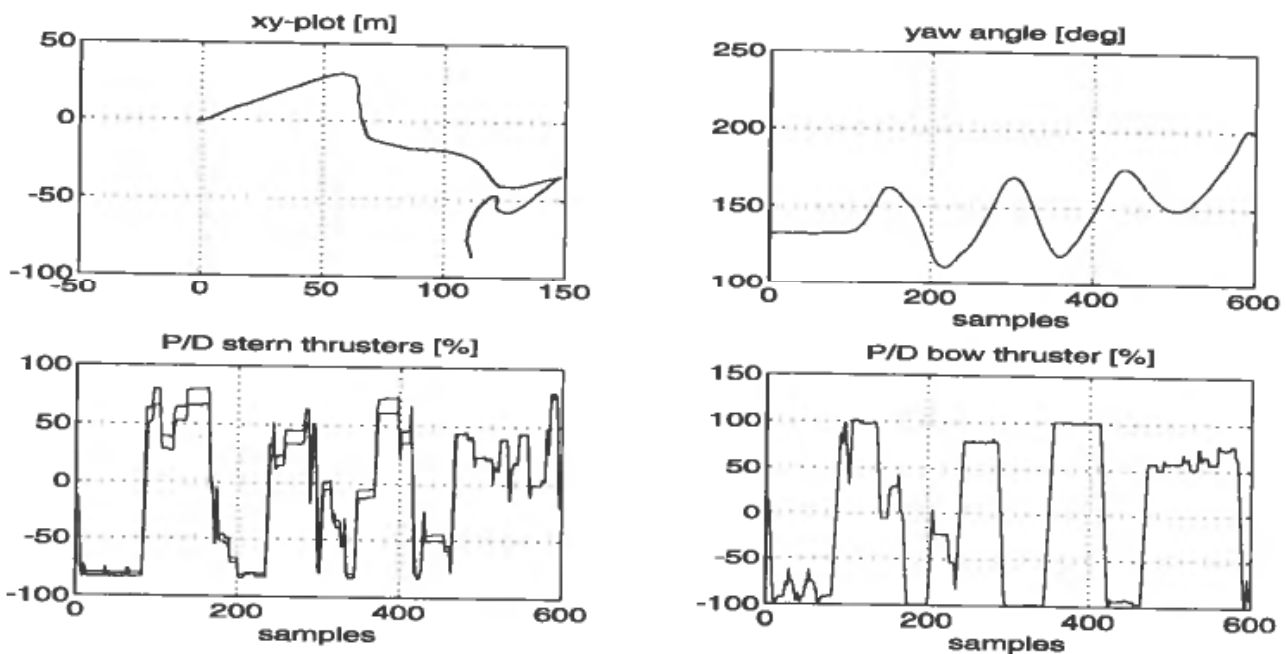

Figure 6. Sea Trial 2: Full scale experiment with a supply vessel (coupled sway and yaw).
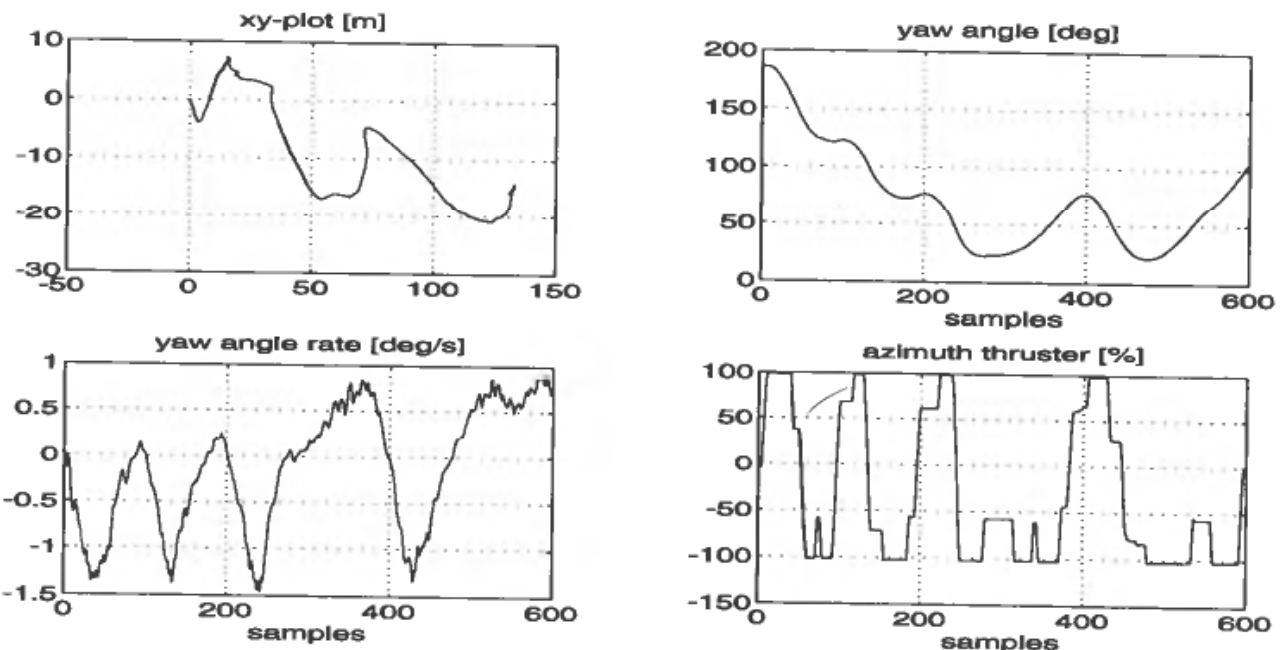

Figure 7. Sea Trial 3: Full scale experiment with a supply vessel (azimuth test).

(2) coupled sway and yaw: the ship should perform two coupled manoeuvres in sway and yaw by means of the three tunnel thruster $u_{3}, u_{4}$ and $u_{5}$. Two manoeuvres should be performed; see Fig. 6.

(3) azimuth test: the last test involves running the azimuth thruster $u_{6}$ alone. Two measurements series are required; see Fig. 7.

\subsection{Implementation issues}

This implies that at least 6 sea trials must be performed for $N=2$. The first two sea trials are used to identify the parameters $K_{1}^{\prime \prime}=K_{2}^{\prime \prime}$ and $X_{u}^{\prime \prime}$ in the decoupled surge equation

$$
\begin{aligned}
\left(1-X_{\dot{u}}^{\prime \prime}\right) \dot{u}^{\prime \prime}-X_{u}^{\prime \prime} u^{\prime \prime} & =K_{1}^{\prime \prime} u_{1}^{\prime \prime}+K_{2}^{\prime \prime} u_{2}^{\prime \prime} \\
\dot{x}^{\prime \prime} & =u^{\prime \prime}
\end{aligned}
$$

where $X_{\dot{u}}^{\prime \prime}$ is computed by using strip theory (Faltinsen 1990). The parameter vector 
corresponding to this system is denoted as $\theta_{1}^{\prime \prime}=\left[K_{1}^{\prime \prime}, X_{u}^{\prime \prime}\right]^{\mathrm{T}}$. The estimated parameter vector $\hat{\boldsymbol{\theta}}_{1}^{\prime \prime}$ in surge is frozen and used as input for the second system identification scheme (S12), that is the coupled sway and yaw identification. Similarly, the output from the second parameter estimation scheme $\hat{\boldsymbol{\theta}}_{2}^{\prime \prime}$ is frozen and used as input for the last parameter estimation scheme (SI3), that is $\hat{\boldsymbol{\theta}}_{3}^{\prime \prime}$. The last scheme is used to estimate only one parameter, that is $\hat{\theta}_{3}^{\prime \prime}=K_{6}^{\prime \prime}$ whereas the second parameter estimation scheme is used to estimate the coupling terms in sway and yaw; see Fig. 4. In the last to parameter estimation schemes the ship is commanded to change heading during the manouvres which implies that the nonlinear kinematic equation

$$
\dot{\eta}^{\prime \prime}=J^{\prime \prime}\left(\eta^{\prime \prime}\right) v^{\prime \prime}
$$

where $\eta^{\prime \prime}=\left[x^{\prime \prime}, y^{\prime \prime}, \psi^{\prime \prime}\right]^{\mathrm{T}}$ should be used together with the dynamic model (26). Hence the unknown parameter vector corresponding to sea trials 2 is $\boldsymbol{\theta}_{2}^{\prime \prime}=\left[Y_{v}^{\prime \prime}, Y_{r}^{\prime \prime}, N_{v}^{\prime \prime}, N_{r}^{\prime \prime}, K_{3}^{\prime \prime}, K_{5}^{\prime \prime}\right]^{\mathrm{T}}$. In this example the tunnel thrusters at the stern are of same type $\left(K_{4}^{\prime \prime}=K_{3}^{\prime \prime}\right)$. It is convenient to rewrite (26) and (33) in terms of the vessel momentum

$$
h^{\prime \prime}=M^{\prime \prime} v^{\prime \prime}
$$

and a momentum bias term $\boldsymbol{b}_{h}$ which yields the following model

$$
\begin{aligned}
\boldsymbol{h}^{\prime \prime}+\boldsymbol{C}_{h}\left(\boldsymbol{h}^{\prime \prime}\right) \boldsymbol{h}^{\prime \prime} & =\boldsymbol{A}_{h}^{\prime \prime}\left(\boldsymbol{\theta}^{\prime \prime}\right) \boldsymbol{h}^{\prime \prime}+\boldsymbol{T}^{\prime \prime} \boldsymbol{K}^{\prime \prime}\left(\boldsymbol{\theta}^{\prime \prime}\right) \boldsymbol{u}^{\prime \prime}+\boldsymbol{b}_{h}+\boldsymbol{w}_{h} \\
\dot{\boldsymbol{\eta}}^{\prime \prime} & =\boldsymbol{J}^{\prime \prime}\left(\boldsymbol{\eta}^{\prime \prime}\right) \boldsymbol{M}^{\prime \prime-1} \boldsymbol{h}^{\prime \prime} \\
\dot{b}^{\prime \prime} & =\boldsymbol{w}_{b} \\
\dot{\theta}^{\prime \prime} & =\boldsymbol{w}_{\theta}
\end{aligned}
$$

Here $\boldsymbol{w}_{h}, \boldsymbol{w}_{b}$ and $\boldsymbol{w}_{\theta}$ are zero-mean Gaussian white noise processes, $\boldsymbol{b}_{h}$ is a slowly-varying parameter representing unmodeled dynamics and environmental disturbances, $\boldsymbol{\theta}^{\prime \prime}$ is the parameter vector to be estimated. The new matrices in the model are defined according to

$$
\begin{gathered}
\boldsymbol{A}_{\boldsymbol{h}}=-\boldsymbol{D M} \\
\boldsymbol{C}_{h}(\boldsymbol{h})=\boldsymbol{C}\left(\boldsymbol{M}^{-1} \boldsymbol{h}\right)
\end{gathered}
$$

Since $M$ is assumed to be known with sufficient accuracy the only unknown quantities in (35)-(38) are $\boldsymbol{A}_{h}$ and $\boldsymbol{K}$. The main motivation for using the momentum equation instead of the standard dynamic equations of motion is improved performance of the state and parameter estimator. Moreover estimation of the states $\boldsymbol{h}=\boldsymbol{M v}, \boldsymbol{\eta}$ and $\boldsymbol{b}_{h}$ together with the parameter vector $\theta$ is easier to perform than estimation of $v, \eta, b_{h}$ and $\boldsymbol{\theta}$. Hence the resulting model can be written

$$
\begin{aligned}
\boldsymbol{x}_{1}(k+1) & =\boldsymbol{f}\left(\boldsymbol{x}_{1}(k), \boldsymbol{u}_{1}(k), \boldsymbol{\theta}(k)\right)+\boldsymbol{w}_{1}(k) \\
\boldsymbol{x}_{2}(k+1) & =\boldsymbol{f}\left(\boldsymbol{x}_{2}(k), \boldsymbol{u}_{2}(k), \boldsymbol{\theta}(k)\right)+\boldsymbol{w}_{2}(k) \\
\boldsymbol{\theta}(k+1) & =\boldsymbol{\theta}(k)+\boldsymbol{w}_{\theta}(k)
\end{aligned}
$$

where $\boldsymbol{x}_{i}=\left[\boldsymbol{h}_{i}^{\mathrm{T}}, \boldsymbol{\eta}_{i}^{\mathrm{T}}, \boldsymbol{b}_{h i}^{\mathrm{T}}\right]^{\mathrm{T}}, \boldsymbol{w}_{i}=\left[\boldsymbol{w}_{h i}^{\mathrm{T}}, \boldsymbol{w}_{b i}^{\mathrm{T}}\right]^{\mathrm{T}}(i=1,2)$ and with obvious definition of $\boldsymbol{f}$. If position $(x, y)$ and heading $(\psi)$ are measured (23) becomes

$$
\begin{aligned}
& z_{1}(k)=H_{1} x_{1}(k)+v_{1}(k) \\
& z_{2}(k)=H_{2} x_{2}(k)+v_{2}(k)
\end{aligned}
$$



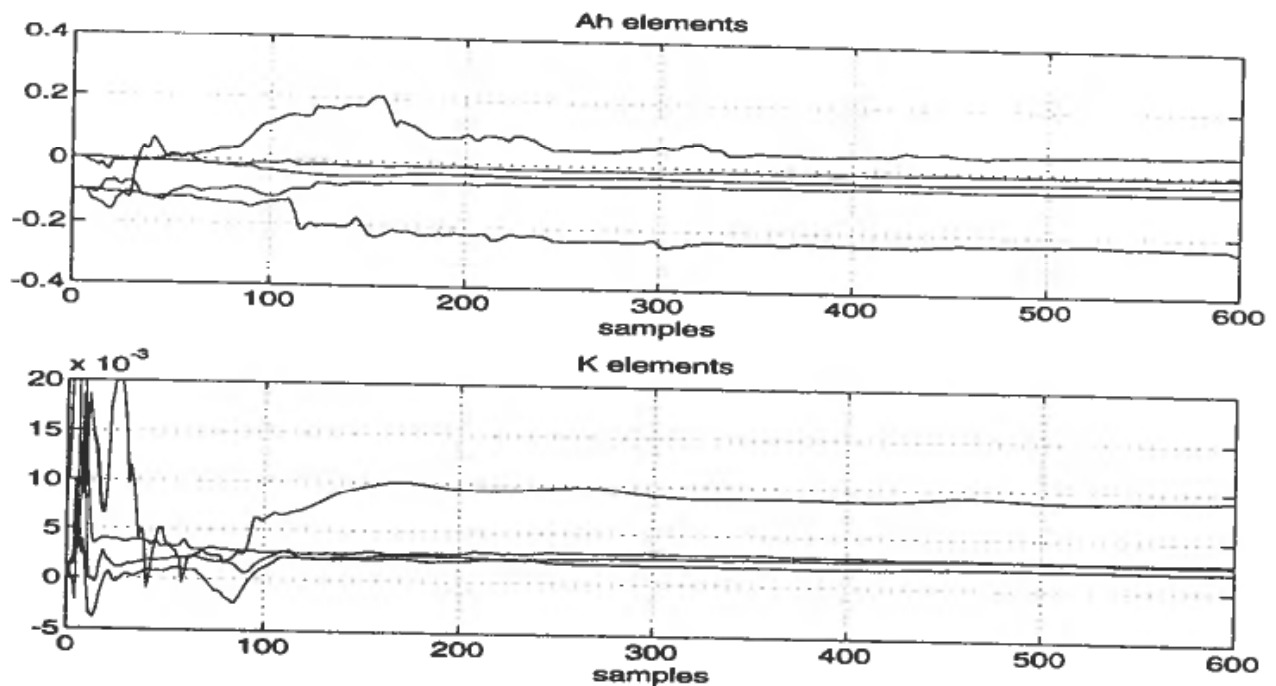

Figure 8. Parameter estimates $\theta_{1}^{\prime \prime} \theta_{9}^{\prime \prime}$ versus time for the supply vessel.

\subsection{Experimental results}

Six manoeuvres with the supply vessel were used to estimate the DP model. The unknown parameter vector $\theta^{\prime \prime}=\left[\theta_{1}^{\prime \prime}, \ldots, \theta_{9}^{\prime \prime}\right]^{\mathrm{T}}$ is organized according to

$$
\begin{aligned}
\boldsymbol{A}_{h} & =\left[\begin{array}{ccc}
\theta_{1}^{\prime \prime} & 0 & 0 \\
0 & \theta_{2}^{\prime \prime} & \theta_{3}^{\prime \prime} \\
0 & \theta_{4}^{\prime \prime} & \theta_{5}^{\prime \prime}
\end{array}\right] \\
\boldsymbol{K}^{\prime \prime} & =\operatorname{diag}\left\{\theta_{6}^{\prime \prime}, \theta_{6}^{\prime \prime}, \theta_{7}^{\prime \prime}, \theta_{7}^{\prime \prime}, \theta_{8}^{\prime \prime}, \theta_{9}^{\prime \prime}\right\}
\end{aligned}
$$

The parameter estimates for the off-line parallel configuration of the EKF algorithm is shown in Fig. 8 whereas the steady-state numerical values are given below.

\section{Identified momentum equation}

$$
\begin{aligned}
& \hat{\boldsymbol{A}}_{h}^{\prime \prime}=\left[\begin{array}{ccc}
-0.0318 & 0 & 0 \\
0 & -0.0602 & 0.0618 \\
0 & -0.0075 & -0 \cdot 2454
\end{array}\right] \\
& \hat{\boldsymbol{K}}^{\prime \prime}=10^{-3} \operatorname{diag}\{9 \cdot 3,9 \cdot 3,2 \cdot 0,2 \cdot 0,2 \cdot 8,2 \cdot 6\}
\end{aligned}
$$

Identified state-space model. The estimated model (35) can be related to the DP control model by assuming that $\boldsymbol{C}_{h}(\boldsymbol{h})=\mathbf{0}$. Hence

$$
\dot{v}^{\prime \prime}=\boldsymbol{A}^{\prime \prime} \boldsymbol{v}^{\prime \prime}+B \tau^{\prime \prime}
$$


where $\boldsymbol{A}^{\prime \prime}=\boldsymbol{M}^{\prime \prime-1} \hat{A}_{h}^{\prime \prime} \boldsymbol{M}^{\prime \prime}$ and $\boldsymbol{B}^{\prime \prime}=\boldsymbol{M}^{\prime-1}$. The numerical values are

$$
\begin{aligned}
\hat{\boldsymbol{A}}^{\prime \prime} & =\left[\begin{array}{cccc}
-0.0318 & 0 & 0 \\
0 & -0.0628 & -0.0030 \\
0 & -0.0045 & -0.2428
\end{array}\right] \\
\hat{\boldsymbol{B}}^{\prime \prime} & =\left[\begin{array}{rrrrrl}
0.0082 & 0.0082 & 0 & 0 & 0 & 0 \\
0.0001 & -0.0001 & 0.0008 & 0.0008 & 0.0020 & 0.0017 \\
0.0035 & -0.0035 & -0.0059 & -0.0055 & 0.0113 & 0.0079
\end{array}\right]
\end{aligned}
$$

For more details about the DP control system design, see Sørensen et al. (1995).

\section{Conclusions}

In this paper a new approach for identification of dynamically positioned ships has been proposed. Three different ship manoeuvres were used in a decoupled identification scheme based on an off-line parallel configuration of the extended Kalman filter algorithm. Simulation studies showed that the proposed parameter estimation scheme was remarkably accurate for ship models that were coupled in surge, sway and yaw. The parameter estimation algorithm has been implemented and tested on a supply vessel. The estimated model of the supply vessel has been implemented and used for model-based DP control system design. The estimated values of this ship showed good agreement with experimental results from model tests.

\section{REFERENCES}

AвкоwiтZ, M. A. (1975). System identification techniques for ship maneuvering trials. In: Proceedings of Symposium on Control Theory and Navy Applications. Monterey, CA. pp. 337-393.

AвKowITZ, M. A. (1980). Measurement of hydrodynamic characteristics from ship maneuvering trials by system identificatior. In: Transactions on SNAME, 88, 283-318.

BALCHEN, J. G.,JENSSEN, N. A., and S ELLID, S. (1976). Dynamic positioning using Kalman filtering and optimal control theory. In: IFACIIFIP Symposium on Automation in Offshore Oil Field Operation. Holland, Amsterdam. pp. 183-186.

BALCHEN, J. G., JENSSEN, N. A., and SÆLID, S. (1980a). Dynamic positioning of floating vessels based on Kalman filtering and optimal control. In: Proceedings of the 19th IEEE Conference on Decision and Control. New York, NY. pp. 852-864.

Balchen, J. G., Jenssen, N. A., Mathisen, E., and S flid, S. (1980b). Dynamic positioning system based on Kalman filtering and optimal control. Modeling, Identification and Control, 1, 135-163.

FALTINSEN, O. M. (1990). Sea Loads on Ships and Offshore Structures (Cambridge University Press).

FosSEN, T. I. (1994). Guidance and Control of Ocean Vehicles (John Wiley and Sons Ltd).

FunG, P. T.-K., and GrIMBLE, M. J. (1983). Dynamic ship positioning using a self tuning Kalman filter. IEEE Transactions on Automatic Control, 28, 339-349.

Gelb, A., Kasper, J. F., JR., Nash, R. A., JR., Price, C. F., and Sutherland, A. A., Jr. (1988). Applied Optimal Estimation (MIT Press, Boston, Massachusetts).

Grimble, M. J., PAtTon, R. J., and Wise, D. A. (1980a). The design of dynamic positioning control systems using stochastic optimal control theory. Optimal Control Applications and Methods, 1, 167-202.

Grimble, M. J., PATton, R. J., and WiSE, D. A. (1980b). Use of Kalman filtering techniques in dynamic ship positioning systems. In: IFE Proceedings. 127, D, 93-102. 
HWANG, WEI-YUAN (1980). Application of System Identification to Ship Maneuvering. Master's thesis. Massachusetts Institute of Technology.

Salid, S., Jenssen, N. A., and BAlChEN, J. G. (1983). Design and analysis of a dynamic positioning system based on Kalman filtering and optimal control. IEEE Transaction on Automatic Control, 28, 331-339.

Sørensen, A., SAgatun, S. I., and Fossen, T. I. (1995). The design of a dynamic positioning system using model based control. In: Preprints IFAC Workshop on Control Applications in Marine Systems (CAMS'95). Trondheim, Norway. 